\title{
El consentimiento informado: recomendaciones para su documentación
}

Miguel Ángel Celis, José Halabe, Oscar Arrieta, Rubén Burgos, Carlos Campillo, Manuel De la Llata, Judith Domínguez, Sergio Islas, Luis Jasso-Gutiérrez, Alberto Lifshitz, Mucio Moreno, Ricardo Plancarte-Sánchez, Alejandro Reyes-Sánchez, Guillermo Ruiz-Argüelles, Antonio Soda, Emma Verástegui y Julio Sotelo

Comité de Ética y Transparencia en la Relación Médico-Industria (CETREMI), Academia Nacional de Medicina de México, Ciudad de México, México

\section{Resumen}

El consentimiento informado es un elemento indispensable para obtener la adecuada participación del paciente, ya sea en protocolos de investigación o en diseños terapéuticos. El Comité de Ética y Transparencia en la Relación Médico-Industria (CETREMI) de la Academia Nacional de Medicina elaboró varias recomendaciones para documentar el consentimiento informado.

PALABRAS CLAVE: Derechos Humanos en medicina. Ética médica. Aceptación de tratamiento. Autorización de participación. Información al enfermo. Medicina defensiva.

\begin{abstract}
Informed consent is an indispensable element to obtain adequate patient participation either in research protocols or in therapeutic design. The Committee of Ethics and Transparency in the Physician-Industry Relationship (CETREMI) of the National Academy of Medicine developed several recommendations for informed consent to be documented.
\end{abstract}

KEY WORDS: Human rights in Medicine. Medical ethics. Treatment acceptance. Participation authorization. Patient information. Defensive medicine.

El documento denominado consentimiento informado es esencial en la medicina moderna como una manifestación de respeto para la dignidad e igualdad del enfermo ante el ejercicio de los profesionales de la medicina y la investigación.

Considerando la enorme relevancia del consentimiento informado, el Comité de Ética y Transparencia en la Relación Médico-Industria (CETREMI) de la Academia Nacional de Medicina opina que el documento de consentimiento informado debe:

1. Reconocer la igualdad humana en sus principios y valores. El consentimiento informado es fundamentalmente un acto humano de igualdad (el paciente) frente a otro ser humano (el médico), dejando atrás la actitud anacrónica de paternalismo médico, que pretende situar al médico por encima del paciente.

2. Aceptar que el consentimiento informado es un acto médico que va más allá de la declaración de riesgos-beneficios. En la práctica diaria constituye un documento de notificación de procedimientos diagnósticos o de tratamientos invasivos necesarios o benéficos, pero que representan un potencial riesgo o daño para el paciente.
Fecha de recepción: 16-04-2018

Fecha de aceptación: 23-04-2018

DOI: 10.24875/GMM. 18004339
Gac Med Mex. 2018;154:716-718

Disponible en PubMed

www.gacetamedicademexico.com 
3. Revalorizar el acto de consentimiento informado que en la rutina diaria en numerosos casos se ha devaluado o desvirtuado utilizándolo primordialmente como instrumento de defensa legal ante posibles reclamaciones 0 demandas.

4. Reforzar los alcances éticos al que debe aspirar el acto médico en términos de igualdad, dignidad, autonomía y respeto de la persona.

5. Representar la mejor propuesta de diagnóstico o de procedimiento terapéutico, de acuerdo con la medicina basada en evidencia científica, en algoritmos y guías clínicas de manejo.

6. Admitir que el paciente es primero y que es una obligación no hacerlo sufrir inútilmente, no violar su dignidad, evitar riesgos adicionales y la obstinación terapéutica o beneficios financieros adicionales, argumentando intereses educativos, de investigación o de innovación.

7. Promover entre los médicos y las instituciones de salud la transparencia y rendición de cuentas. Es necesario identificar o prevenir conflictos derivados de intereses particulares, por iniciativa de la industria farmacéutica o de los proveedores de insumos o equipos biomédicos al momento de realizar el consentimiento informado. En los procedimientos por autorizar solo deberá utilizarse lo necesario y evitar lo superfluo.

8. Documentar si las decisiones derivaron de una reunión médica, colegiada o de consenso. Si se siguen modelos o guías internacionales se debe informar al paciente que la implementación práctica también es válida o comparable en su propia experiencia o la de la institución. Promover la discusión clínica colegiada y documentar las decisiones o propuestas de manejo que apoyen el consentimiento.

9. Promover entre los médicos y las instituciones cursos de capacitación para la obtención del consentimiento informado, enfatizando el trato digno y aceptación de la identidad de la persona (paciente), lo que implica otorgar al enfermo un trato como iguales, ni superiores ni inferiores; y aceptar sin prejuicios las diferencias por religión, etnicidad, género, clase social, nivel educativo, orientación sexual, edad o discapacidad.

10. Asegurar el balance entre la cantidad y calidad de información a definir en el consentimiento: a) de acuerdo con la urgencia del caso (a mayor urgencia, menor precisión exigible en la información), b) la necesidad de tratamiento (si la intervención es menos necesaria, mayor rigor para sustentarla, que debe ser extremo en el caso de la cirugía estética), c) la peligrosidad o alto riesgo de complicaciones, d) la novedad del procedimiento, e) la gravedad de la enfermedad (balance juicioso entre riesgo-beneficio).

11. Ante la negativa del paciente a aceptar procedimientos necesarios por carencias económicas, el médico debe orientarlo a buscar otras opciones médico-quirúrgicas en los establecimientos públicos.

Es opinión del CETREMI que se debe evitar:

a) Ser demasiado explícito y causar depresión, angustia o miedo en los pacientes al conocer su diagnóstico, si este no fuera tan bueno como se esperaba.

b) Que el paciente se sienta inseguro y cambie de médico por otro que le plantee un panorama más optimista, no necesariamente más real, con menor ética y posiblemente menos competente.

c) Que en las instituciones públicas o privadas, médicos con poca experiencia obtengan el consentimiento informado. Evitar las prácticas rutinarias, inerciales o históricas.

d) Informar en forma indebida al paciente o familiares, para inducirlos a la aceptación de un procedimiento no necesario o que implique riesgo excesivo para el padecimiento que se pretende atender, con objeto de adquirir experiencia para "integrar un caso", con fines de presentación académica o publicación, para protegerse de posibles quejas o demandas (medicina defensiva), con propósitos comerciales o de beneficio económico.

e) Informar en forma malintencionada al paciente 0 a los familiares para generarles miedo, buscando su disentimiento, para eludir la práctica de un procedimiento médico o quirúrgico, diagnóstico o terapéutico, en especial en hospitales públicos.

f) Dar información alarmante dirigida a "cubrirse" (medicina defensiva) de posibles quejas, demandas, riesgos, eventualidades adversas, no prevenibles, riesgos de complicación, impericia, incompetencia o mala práctica ante posibles resultados adversos, cuando desee deshacerse del enfermo con el propósito de que el paciente no sea demandante o para que busque a otro médico. 
g) En las instituciones de medicina privada, que se omita o no sea explícito el documento de consentimiento informado. El documento formal debe ser práctica cotidiana en el ejercicio privado de la medicina. h) CETREMI enfatiza que el documento "consentimiento informado" no tiene por objeto proteger al médico de eventuales demandas y reclamaciones, sino proteger a los pacientes de eventuales abusos u omisiones del personal de salud. 